\title{
THE X-RAY SPECTRA OF HIGH REDSHIFT QUASARS
}

\author{
CLAUDE R. CANIZARES and JULIA L. WHITE \\ Department of Physics and Center for Space Research \\ Massachusetts Institute of Technology \\ 77 Massachusetts Avenue \\ Cambridge, MA 02139 \\ U.S.A.
}

\begin{abstract}
We present mean spectral parameters for various ensembles of quasars observed with the Einstein Observatory Imaging Proportional Counter (IPC). Our sample contains 71 optically or radio selected quasars with $0.1<\mathrm{z}<3.5$, Galactic $\mathrm{N}_{\mathrm{H}}<10^{21}$ $\mathrm{cm}^{-2}$, total counts of $30-500$, and IPC gain $<19$. Quasars are grouped into ensembles according to radio properties (Flat Radio Spectrum [FRS], Steep Radio Spectrum [SRS] or Radio Quiet [RQ]), and either redshift or X-ray luminosity, $l_{\mathrm{x}}$. We find a clear correlation between radio properties and $\alpha$. FRS quasars have $\alpha \sim 0.4$, SRS quasars have $\alpha \sim 0.7$ and RQ quasars have $\alpha \sim 1-1.4$. There is no evidence for a dependence of $\alpha$ on $\mathrm{z}$ nor, for the FRS and SRS ensembles, on $l_{\mathrm{x}}$ over nearly three decades. FRS quasars with $2.0<\mathrm{z}<3.5$ have just as flat mean spectra as those with low $\mathrm{z}$, implying that a single power law, which is flatter than the canonical one with $\alpha \sim 0.65$, continues into the $1-10$ $\mathrm{keV}$ band (in which the observed softer X-rays were emitted). Unfortunately, the results for high redshift and high $l_{\mathrm{x}} \mathrm{RQ}$ quasars are ambiguous because of systematic uncertainties in the ensemble means. Thus we cannot test the two-component spectral hypothesis of Wilkes and Elvis for these objects. SRS X-ray spectra could be steeper than FRS spectra because of the mixing of two components, although a single intrinsically steeper spectrum is easier to reconcile with the absence of $z$ dependence. The uncertainty in $\alpha$ for RQ quasars with high $\mathrm{z}$ leaves open the important question of their contribution to the cosmic X-ray background.
\end{abstract}

\section{INTRODUCTION}

We examine the X-ray spectra of high redshift quasars using data from the Imaging Proportional Counter (IPC) on the Einstein Observatory (Giacconi et al. 1979). In general the objects we wish to study are too faint to permit spectral analysis. So rather than treating them individually, we group them into ensembles and find their mean spectral parameters. We group the samples according to radio properties, redshift, and luminosity. Drawing conclusions about the physics of quasars from our results requires the assumption that within each ensemble the spectral properties of the quasars are similar enough to give meaning to the concept of an average spectrum. The implications for the cosmic X-ray background are independent of such an assumption, but they do require that 
our sample is reasonably representative. A full report of this work has been submitted to the Astrophysical Journal.

\section{THE QUASAR SAMPLE}

Our quasars are drawn from the samples of Tananbaum et al. (1986), Avni and Tananbaum (1986), and Worrall et al. (1987), which include most of the optically and radio selected quasars observed by Einstein. We set four selection criteria: (i) z $>0.1$; (ii) Galactic $\mathrm{N}_{\mathrm{H}}<10^{21} \mathrm{~cm}^{-2}$; (iii) total number of detected photons in the observation $>30$ but $<500$ (the upper limit prevents a few bright objects from dominating and is only relevant for the lowest redshift ensemble); (iv) the IPC instrumental gain during the observation be within an acceptable bound $(<19)$.

Our selected sample contains 71 quasars. The objects are distributed among two major categories, Radio Quiet (RQ) and Radio Loud (RL). The latter objects are further subdivided into Steep Radio Spectrum (SRS) and Flat Radio Spectrum (FRS). These categories follow the conventions of Worrall et al. (1987).

The three major subdivisions have comparable numbers of sources, although those in the RQ sample generally have fewer counts.

\section{DATA ANALYSIS}

Each of the observations listed in Tables 1 and 2 was fit to a simple three parameter power law form, $d N(E) / d E d A d t=I_{0} \exp \left[-N_{H} \sigma(E)\right] E^{-\alpha-1}$, where $d N(E) / d E d A d t$ is the specific flux of photons of energy $E$ at earth, $\sigma(E)$ is the absorption cross section at $E$ in cold material with cosmic abundances (from Brown and Gould 1970), and $\mathrm{I}_{0}, \mathrm{~N}_{\mathrm{H}}$, and $\alpha$ are the parameters to be determined. We determined the best fit parameters for an entire subsample by adding together the individual grids of $\chi^{2}$ vs. $\alpha$ and $\mathrm{N}_{\mathrm{H}}$ to form a composite grid from which one can read off directly the desired information. This is effectively equivalent to performing a single joint fit to the data of all the quasars in the subsample (see our Ap. J. paper for details).

\section{COMPOSITE SPECTRA OF QUASAR SUBSAMPLES}

As noted above, we divided our sample into three categories based on radio properties: RQ, SRS and FRS. Each of these is further separated into three or four subgroups by redshift or by X-ray luminosity (we use $\mathrm{H}_{\mathrm{o}}=50 \mathrm{~km} \mathrm{~s}^{-1} \mathrm{Mpc}^{-1}$ ).

Wilkes and Elvis (1987) established that quasar spectra vary considerably in slope, with RL quasars being significantly flatter than RQ quasars. By segregating our sample according to radio properties, we have attempted to achieve greater homogeneity among the objects that we combine. However, each ensemble is small enough to cause concern that the composite may be dominated by one object with an unrepresentative spectrum. In order to test for this possibility, we examined numerous composite grids for each subgroup with one or more members excluded. More detail on the results of this exercise is given in our Ap. J. paper, but our conclusion is that the composite spectra are reasonably robust for most of the subgroups. Unfortunately, the high redshift RQ sample 
is dominated by one strong source and so our results for this important subgroup are very uncertain.

The $90 \%$ confidence contours, and usually the $65 \%$ confidence contours as well, encompass the value zero for the residual hydrogen column density. Therefore, we conclude that there is no evidence for excess absorption above the galactic value for the objects in our sample. In all the following discussion, we will assume no excess absorption, and all the spectral indices we quote incorporate this assumption.

a)

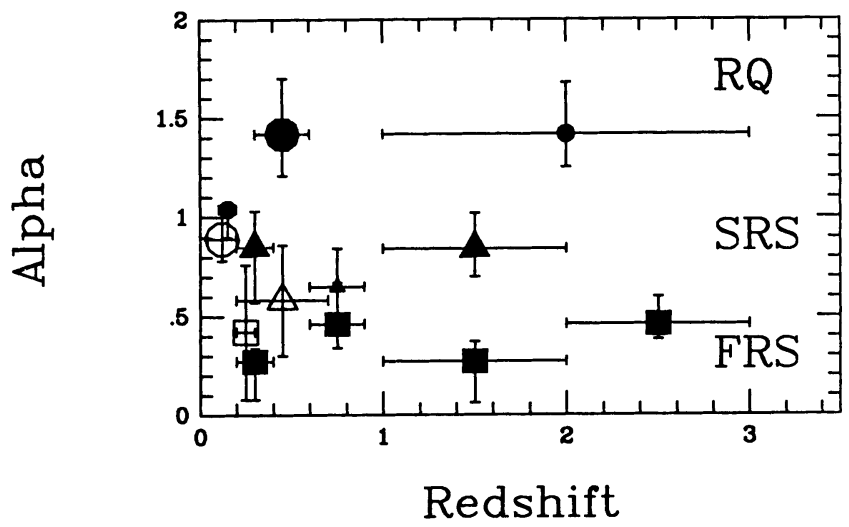

b)

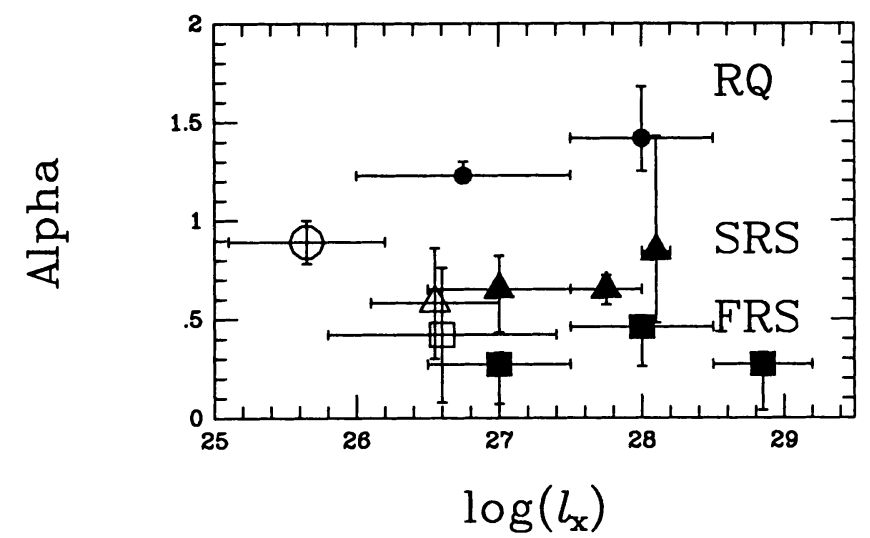

Figure 1. a) Power law index $\alpha$ vs. redshift for the various subsamples as deduced from the composite $\chi^{2}$ contours assuming zero residual $\mathrm{N}_{\mathrm{H}}$. The circles indicate RQ quasars, the triangles SRS quasars and the squares FRS quasars. The horizontal bars indicate the range of redshift for the quasars in each subsample, whereas the vertical bars give the $90 \%$ uncertainty based on the $\chi^{2}$ contours. The smaller points are suspect because of systematic uncertainties as described in the text. The open symbols are taken from the data of Wilkes and Elvis 1987. b) Power law index $\alpha$ vs. X-ray luminosity. The symbols and error bars are as in a). 

as follows:

Our results are given in Figure 1 (see caption for details). They can be summarized

There is a clear correlation between radio properties and $\mathrm{X}$-ray spectral index. FRS quasars have the flattest X-ray spectra, RQ quasars have the steepest, and SRS quasars are intermediate.

The $\mathrm{X}$-ray spectral index for quasars with the same radio properties shows no strong dependence on redshift. RQ quasars with $\mathrm{z}>0.3$ may have somewhat steeper spectra than those at lower redshift ( $\alpha \sim 1.4$ vs. $\alpha \sim 1.0$ ), although this could well be an artifact of the systematic uncertainties associated with the RQ ensemble (our lowest $\mathrm{z}$ value moves higher and our highest $\mathrm{z}$ value moves lower when we exclude the brightest members of the subsets; see Section IVA). Of course any dependence of $\alpha$ on $\mathbf{z}$ would be hard to distinguish from a dependence on $l_{\mathrm{x}}$.

The SRS and FRS quasars show no dependence of $\alpha$ over nearly three decades of $\mathrm{X}$-ray luminosity. For RQ quasars, there is a progressive increase in $\alpha$ with $l_{\mathrm{x}}$, from $\sim 0.9$ to $\sim 1.4$. This is more suggestive than the corresponding trend with redshift, but the same caveats about systematic uncertainties apply (in particular the high $l_{\mathrm{x}}$ and high $\mathrm{z}$ ensembles are identical and are dominated by one object with a large $\alpha$; see section IVA).

For the highest redshift FRS ensemble, the observed energy range corresponds to the range $\sim 1-10 \mathrm{keV}$ at the source. The fact that $\alpha$ for this ensemble is the same as that for low redshift objects suggests that the power law spectrum for FRS quasars extends into the hard X-ray band (in the quasar rest frame). The mean $\alpha$ for all FRS ensembles, including that at the highest redshift, is smaller than the value 0.65 which had long been considered a "magic" number that applied to all AGN spectra in the $1-10 \mathrm{keV}$ band (e.g. Mushotzky 1984).

\section{DISCUSSION}

Our data support the conclusion of Wilkes and Elvis (1987) that RL quasars have significantly flatter X-ray spectra that RQ quasars. Furthermore, we find that within the RL sample, FRS quasars have flatter spectra than SRS quasars, and the flat FRS spectra appear to extend into the hard X-ray band. It is unfortunate that the most interesting data point is not well determined, namely the mean $\alpha$ of high redshift RQ quasars. Formally it is large $(\langle\alpha\rangle \sim 1.4)$, but the ensemble is dominated by one object $(1407+267$ with $z=$ 0.94 ), and when this is removed $\alpha$ is not well constrained (the $90 \%$ limits on $\alpha$ are 0.3 1.4 and the $68 \%$ limits are $0.5-1.2$ ). Thus the question of whether the steeper power-law spectra of RQ quasars also extends into the hard X-ray band remains unanswered.

Wilkes and Elvis suggest that there are two distinct components that contribute to the X-ray spectra of quasars, one with $\alpha \sim 1$, which is associated with the optical/IR emission and is present in all quasars, and one with $\alpha \sim 0.5$, which is associated with the radio emission. They hypothesize that the "magic index" $\alpha \sim 0.65$ found in earlier studies for all AGN (including some that are radio quiet, e.g. Mushotzky 1984) results from an accidental mix of these two components at energies of $\sim 1-2 \mathrm{keV}$. At higher energies the 
flatter power law should dominate for all objects, including the RQ quasars. Because of the ambiguity of the high $\mathrm{z} R \mathrm{R}$ data point, our results neither confirm nor do they contradict this hypothesis.

The intermediate values of the spectral indices of SRS quasars might indicate that mixing of these same components occurs at lower energies for these objects than for RQ quasars. This follows if the flatter component is relatively stronger in SRS than in RQ quasars, although not as strong as in FRS objects, for which it dominates over most or all of the IPC band. This would be qualitatively consistent with the attempt of Worrall et al. (1987) to separate the fractional contributions to $\mathrm{L}_{\mathrm{x}}$ from components related to the coreradio and to the optical emission; on average the fractional contribution of the radio related X-ray flux is smaller for SRS quasars (with extended morphology) than for FRS quasars. On the other hand, the mixing hypothesis for SRS quasars is not consistent with our finding that their spectral indices are independent of $\mathrm{z}$ and therefore of shifts in energy passband by up to a factor of two; one would have expected the higher $z$ objects to have flatter spectra. A possible alternative to the mixing hypothesis is that the radiorelated X-ray component in SRS quasars is simply steeper than in FRS quasars. This is plausible for the inverse-Compton mechanism that is often invoked for this emission (e.g. Worrall et al. 1987, Wilkes and Elvis 1987, Urry 1988).

We emphasize that all these remarks refer to ensemble mean spectral indices. Within any ensemble there is likely to be a distribution of $\alpha$ values about the mean. This is evident particularly for radio quiet quasars, (although some of the spread may come from the ultrasoft component below $0.3 \mathrm{keV}$; Wilkes and Elvis 1987). It is also suggested by our individual fits to FRS quasars, a number of which have $90 \%$ confidence intervals that do not overlap. Elucidation of this point requires considerably better data, however.

What are the implications for the X-ray background? Maccacaro et al. (1988) show that the mean spectral index of the Einstein Medium Survey sources is $\sim 1$. Our study is the first that attempts to constrain the spectral parameters of objects at higher redshifts that give the greatest contribution to the background. The apparent lack of dependence of $\alpha$ on redshift for RL quasars validates what has been assumed by most authors (see Fabian 1988 and references therein). Future analyses should also distinguish the properties of SRS from FRS quasars. But RL quasars contribute at most $10 \%$ to the X-ray background (Kembhavi and Fabian 1982). Our ambiguous conclusions regarding the spectrum of the much more numerous high redshift RQ quasars leaves open the question of their role in forming the X-ray background and fails to resolve the uncertainty over the spectral shape of any residual contributors (Fabian 1988). We must wait for future X-ray missions to provide the answers.

We thank John Kruper, G. Fabbiano and D. Fabricant for assistance with the analysis, and we thank M. Elvis and D. Worrall for helpful conversations. This work was supported in part by NASA NAG-8-494. 


\section{REFERENCES}

Avni, Y., and Tananbaum, H. 1986, Ap. J., 305, 83.

Fabian, A. C. 1988 (preprint).

Giacconi, R. et al. 1979, Ap. J., 230, 540.

Kembhavi, A. K. and Fabian, A. C. 1982, M.N.R.A.S., 198, 921.

Maccacaro, T., Gioia, I., Wolter, A., Zamorani, G. and Stocke, J. T., 1988, Ap. J., 326, 680.

Mushotzky, R.F., 1984, Adv. Space Res., 3, 157.

Tananbaum, H., Avni, Y., Green, R.F., Schmidt, M., and Zamorani, G. 1986, Ap. J., 305, 57.

Urry, M. 1988, in Cordova, F. (ed.), Multiwavelength Astrophysics, (in press).

Wilkes, B., and Elvis, M. 1987, Ap.J., 323, 243.

Worrall, D.M., Giommi, P., Tananbaum, H., and Zamorani, G. 1987, Ap. J., 313, 596. 